Milena BOGDANOVIĆ

УДК 514.113.6

Marko STANKOVIĆ

511.84

Marija JORDANOVIĆ

- оригинални научни рад -

Pedagogical faculty in Vranje

University of Niš

\title{
SOME COMMENTS ON ISOMETRIES OF SPHERE $\mathbb{S}^{2}$
}

\begin{abstract}
The aim of the paper is to show that isometries of sphere are restrictions of isometries in $\mathbb{R}^{3}$, and it is a consequence of the reflections of large circles. This paper presents a modified proof of the theorem of three reflections in $\mathbb{S}^{2}$ and the classification of isometries in $\mathbb{S}^{2}$. The group of isometries of sphere $\mathbb{S}^{2}$ is "very large"; that confirms the theorem of 2-transitivity of sphere. The proof of this theorem is given in the paper. The group of rotations of sphere is especially emphasized and it is shown that the product of any two rotations in $\mathbb{S}^{2}$ is rotation, too. At the end, the paper contains interesting examples of application of isometries of sphere.
\end{abstract}

Key words: Isometries of sphere $\mathbb{S}^{2}$, plane reflection, 2-transitivity of sphere, group of rotations of sphere.

\section{Introduction}

The unit sphere in $\mathbb{R}^{3}$ consists of all points on the unit distance from point $O$; these are all points $(x, y, z)$ that satisfy the equation $x^{2}+y^{2}+z^{2}=1$. This sur-face is also called 2-sphere, or $\mathbb{S}^{2}$, because the points of sphere may be represented by two coordinates - width and height, for example. That geometry is essentially two-dimensional, as the Euclidean plane $\mathbb{R}^{2}$ or the real projective plane $\mathbb{R P}^{2}$, and indeed, the basic objects of spherical geometry are "points" (regular point on the sphere) and "lines" (big circles on the sphere). A distance $\overparen{X Y}$ between points of the sphere $X$ and $Y$ is defined as $\overparen{X Y}=\alpha, \cos \alpha=$ $\langle\overrightarrow{O X}, \overrightarrow{O Y}\rangle, 0 \leq \alpha \leq \pi$. The next theorem from (Blažić, Bokan, Lučić \& Rakić, 2003) holds in spherical geometry:

Theorem 1 The function $\Omega: \mathbb{S}^{2} \times \mathbb{S}^{2} \rightarrow[0, \pi]$ is inner metric on $\mathbb{S}^{2}$. If $X, Y$ are points of sphere $\mathbb{S}^{2}$, such that $X \neq Y$, than there is the unique shortest curve that connects $X$ and $Y$. It is a shorter arc of the great circle, that passes through the points $X$ and $Y$. If $X=-Y$, the shortest curve from $X$ to $Y$ is great circle with ends $X,-X$. 


\section{The isometries}

First we introduce definition of isometric transformation in the same manner as (Lopandić, 2011).

Definition 1 The isometric transformation or geometric motion of the space $E^{n}(n=1,2,3)$ is a bijective transformation $f: E^{n} \rightarrow E^{n}$ so that for each two points $X, Y \in E^{n}$ and their images $X^{\prime}, Y^{\prime} \in E^{n}$ the following relation is $\operatorname{valid}(X, Y) \cong\left(X^{\prime}, Y^{\prime}\right)$.

Therefore, if $f$ is an isometry that fixes point $O$, than $f$ maps all of the points on distance 1 from $O$ into other points on the same distance from $O$. In other words, isometry $f$ in $\mathbb{R}^{3}$ that fixes $O$ maps $\mathbb{S}^{2}$, into itself. A restriction of isometry $f$ in $\mathbb{S}^{2}$, is isometry in $\mathbb{S}^{2}$, because $f$ keeps the distance on $\mathbb{S}^{2}$, as in $\mathbb{R}^{3}$. Isometries in $\mathbb{S}^{2}$, are functions of $\mathbb{S}^{2}$, into itself that keep the distance on the great circle.

The simplest isometries in $\mathbb{R}^{3}$ with a fixed point $O$ are reflections in a plane through $O$. Corresponding isometries in $\mathbb{S}^{2}$ are reflections in the great circles. The product of reflections $\mathcal{S}_{\pi_{1}}$ and $\mathcal{S}_{\pi_{2}}$ is rotation about an axis $l$ (for double angle between $\pi_{1}$ and $\pi_{2}$ ), wherein $l$ is an intersection of the planes $\pi_{1}$ and $\pi_{2}$. This situation is completely analogous to that in $\mathbb{R}^{2}$ where the product of reflections through $O$ is rotation (for double angle between lines).

Finally, those are products of three reflections in planes which are different from products of one or two reflections in planes. One of those isome-tries is antipodal map that maps each point $(x, y, z)$ into itself antipodal point $(-x,-y,-z)$. The following theorem shows why all isometries in $\mathbb{S}^{2}$ are restrictions of isometries in $\mathbb{R}^{3}$. Namely, this is true because of the reflections in great circles. Reflection in a great circle in $\mathbb{S}^{2}$ corresponds to reflection in a plane in $\mathbb{R}^{3}$.

Theorem 2 (Three Reflections Theorem in $\mathbb{S}^{2}$ ) Any isometry of $\mathbb{S}^{2}$ is the com-position of one, two or three reflections in great circles.

Proof 1 Let $f$ be an arbitrary isometry of sphere and $A, B, C$ three "non-collinear" points on sphere, and let $A_{1}, B_{1}, C_{1}$ be images of those points in isometry $f$, respectively. We distinguish the following cases.

Case 1 If $A=A_{1}, B=B_{1}, C=C_{1}, f$ is identity so, if a is an arbitrary line, then $f=\mathcal{S}_{a} \mathcal{S}_{a}$. Therefore, suppose that points $A, B, C$, are not invariant in isometry $f$ but one of them, for example $A$, maps by isometry $f$ into some point on the sphere differente from $A$. If $\mathcal{S}_{1}$ is reflection which maps $A$ into $A_{1}$, then $A$ is invariant point in composition $\mathcal{S}_{1} f$. If $B, C$, are invariant points in that composition, then $f=\mathcal{S}_{1}$.

Case 2 Suppose that in isometry $\mathcal{S}_{1} f$ at least one of the points $B, C$, for example $B$, isn't invariant but $B$ maps into $B_{2}$, and $C$ into $C_{2}$. Let $\mathcal{S}_{2}$ be the reflection in a line which maps $B$ into $B_{2}$. In that case

$$
(A, B) \cong\left(A_{1}, B_{1}\right) \cong\left(A, B_{2}\right)
$$

so point $A$ belongs to basis $a_{2}$ of the reflection $\mathcal{S}_{2}$. Therefore, points $A$ and $B$ are invariant in composition $\mathcal{S}_{2} \mathcal{S}_{1} f$. If $C$ is invariant point, that composition is identity so $f=\mathcal{S}_{1} \mathcal{S}_{2}$. 
Case 3 Suppose that in isometry $\mathcal{S}_{2} \mathcal{S}_{1} f$ point $C$ isn't invariant, but it maps into $C_{3}$. Let $\mathcal{S}_{3}$ be reflection which maps $C$ into $C_{3}$. In that case

$$
\begin{gathered}
(A, C) \cong\left(A_{1}, C_{1}\right) \cong\left(A, C_{2}\right) \cong\left(A, C_{3}\right) \\
(B, C) \cong\left(B_{1}, C_{1}\right) \cong\left(B_{2}, C_{2}\right) \cong\left(B, C_{3}\right),
\end{gathered}
$$

so points $A$ and $B$ belong to the basis $a_{3}$ of the reflection $\mathcal{S}_{3}$. Therefore, points $A, B, C$ are invariant in composition $\mathcal{S}_{3} \mathcal{S}_{2} \mathcal{S}_{1} f$, so $f=\mathcal{S}_{1} \mathcal{S}_{2} \mathcal{S}_{3}$.

The proof of the theorem is completed.

The importance of reflections is reflected in the fact that each isometry of a line, plane, or space can be expressed as composition of finite number of reflections. The following theorems from (Lučić, 1997) are held in $\mathbb{R}^{3}$ :

Theorem 3 Each isometry of a line can be expressed as composition of maximum two reflections in lines.

Theorem 4 Each isometry of a plane can be expressed as composition of maximum three reflections in lines.

Theorem 5 Each isometry of a space can be expressed as composition of maxi-mum four reflections in planes.

All of isometries of space can be divided onto direct (composition of even number of reflections) and indirect (composition of odd number of reflections). Direct isometries of space are: coincidence, translation, rotation about an axis, screw displacement. Indirect isometries of space are: reflection in a plane, improper rotation, glide reflection. Based on the previous, we can perform the classification of isometries of sphere. Direct isometries of sphere $\mathbb{S}^{2}$ ) are:

- coincidence (because $f(O)=O$ where $O$ is center of sphere, and $f$ is coincidence)

- rotation about an axis (composition of two reflections in planes that contain center of sphere $O$. Whereas point $O$ belongs to both planes it follows that $O$ belongs to their intersection i.e. point $O$ belongs to the axis of rotation so $f(O)=O$ ).

Translation and screw displacement don't have fixed points so these isometries aren't isometries of sphere.

Indirect isometries of sphere $\mathbb{S}^{2}$ are:

- reflection in a plane (plane contains the center of sphere $O$, so $f(O)=O)$

- improper reflection (the composition of three reflections in planes; each of the planes contains the center of sphere O. In the special case when all planes are mutually perpendicular, their intersection is center of sphere and that isometry is symmetry of the space). of sphere.

The glide reflection has no fixed points so this isometry isn't isometry

Theorem 6 If an isometry in $\mathbb{R}^{2}$ has more than one invariant point, it must be either the identity or a reflection.

The previous theorem is taken from (Coxeter, 1969). Considering the fact that the geometry of sphere is two-dimensional, analogous theorem holds in $\mathbb{S}^{2}$. 


\section{The transitivity of group of isometries}

The previous facts raise the question of "size of the group of isometries of sphere $\mathbb{S}^{2}$ ". A suitable notion for this question is the transitivity of group of transformations. We will say that a group of isometries $\operatorname{Isom}(\mathcal{M})$, of some set $\mathcal{M}$, is transitive, if for two arbitrary points $A$ and $B$ from $\mathcal{M}$ there is an isometry $f: \mathcal{M} \rightarrow \mathcal{M}$, so that $f(A)=B$. For example, the group of isometries of sphere $\mathbb{S}^{2}$ is transitive, because for any two points of sphere $A$ and $B$, there is a relation of space which axis of rotation is perpendicular to the plane determined by points $O, A$, and $B$ and which translates point $A$ into point $B$. Therefore, this rotation is actually rotation in the plane determined by points $O, A$, and $B$ because two points of axis of rotation are fixed in this rotation.

Analogously we also introduce the following notion. We say that group of isometries of set $\mathcal{M}$ is 2-transitive if for each two pairs of points $A, B$ and $A_{1}, B_{1}$ which are on the same distances (i.e. if $d(A, B)=d\left(A_{1}, B_{1}\right)$ ), there is an isometry $f: \mathcal{M} \rightarrow \mathcal{M}$, which maps $A$ into $A_{1}$, and $B$ into $B_{1}$.

For example, affine space is one example of the spaces with 2-transitive group of isometries.

Theorem 7 (2-transitivity of sphere.) Let $A, B, A_{1}$ and $B_{1}$ be four points of the sphere $\mathbb{S}^{2}$ so that $\overparen{A B}=\overparen{A_{1} B_{1}}$. Then there is an isometry $f \in$ $\operatorname{Isom}\left(\mathbb{S}^{2}\right)$, so that $f(A)=A_{1}$ and $f(B)=B_{1}$.

Proof 2 Let $f_{1}: \mathbb{S}^{2} \rightarrow \mathbb{S}^{2}$ be rotation which maps point $A$ into $A_{1}$. Then

$$
\overparen{A_{1} f_{1}(B)}=\overparen{f_{1}(A) f_{1}(B)}=\overparen{A B}=\overparen{A_{1} B_{1}} \text {, }
$$

so points $f_{1}(B)$ and $B_{1}$ are on the same distance from $A_{1}$. Thus there is rotation $f_{2}: \mathbb{S}^{2} \rightarrow \mathbb{S}^{2}$, so that $f_{2}\left(A_{1}\right)=A_{1}, f_{2}\left(f_{1}(B)\right)=B_{1}$. The required isometry $f$ is the composition of rotations $f_{1}$ and $f_{2}$. (See Figure 1.)

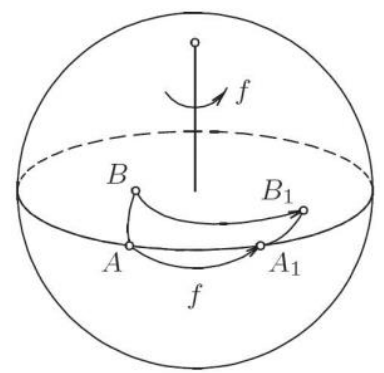

Figure 1: 2-transitivity of sphere

The results of the previous theorem shows that the group of isometries of sphere $\mathbb{S}^{2}$ is very "large", apropos that the sphere $\mathbb{S}^{2}$ is "very simetric" (Blažić et all, 2003). 


\section{The group of rotations of sphere}

The group $\operatorname{Isom}\left(\mathbb{S}^{2}\right)$ of all isometries of sphere $\mathbb{S}^{2}$ has subgroup Isom ${ }^{+}\left(\mathbb{S}^{2}\right)$ consisting of isometries that are product of an even number of reflections. As Isom ${ }^{+}\left(\mathbb{R}^{2}\right)$ this subgroup keeps an orientation. In contrast to Isom ${ }^{+}\left(\mathbb{R}^{2}\right)$, Isom ${ }^{+}\left(\mathbb{S}^{2}\right)$ this subgroup does not include translation, but only rotations. We have already known that the product of two reflections of $\mathbb{S}^{2}$ is a rotation. Hence, in order to show that the product (composition) of an even number of reflections is rotation, it remains to show the following proposition:

Proposition 1 The product (composition) of any two rotations of $\mathbb{S}^{2}$ is a rotation.

Proof 3 Suppose that we have a product of two rotations on $\mathbb{S}^{2}$ :

- rotation for angle $\alpha$ around point $A$ (it is rotation about an axis through point $A$ and diametrically opposite point $-A$ ),

- rotation for angle $\beta$ around point $B$.

Rotation for an angle $\alpha$ around point $A$ is the product of reflections whose axis are "lines" (great circles) through A. Moreover, they can be any "lines" $a$ and $b$ through $A$ until the angle between $a$ and $b$ is equal $\alpha / 2$. Specifically, we can choose a line $b$ so that line passes through points $A$ and B. Similarly, rotation for an angle $\beta$ around point $B$ is the product of reflections in lines of any two lines through $B$ which form an angle $\beta / 2$, so that the first axis can be the "line" $b$. Other axis of reflection through $B$ is therefore the "line" $c$ which forms angle $\beta / 2$ with the "line" $b$. (See Figure 2.)

If $\mathcal{R}_{a}, \mathcal{R}_{b}, \mathcal{R}_{c}$, represent the reflections which axes are $a, b, c$, respectively, then rotation for angle $\alpha$ around $A$ is the composition $\mathcal{R}_{b} \mathcal{R}_{a}$, and rotation for angle $\beta$ around $B$ is the composition $\mathcal{R}_{c} \mathcal{R}_{b}$. Therefore, the product of these rotations is

$$
\mathcal{R}_{c} \mathcal{R}_{b} \mathcal{R}_{b} \mathcal{R}_{a}=\mathcal{R}_{c} \mathcal{R}_{a},
$$

because $\mathcal{R}_{b} \mathcal{R}_{b}$ is identity (Stillwel, 2000).

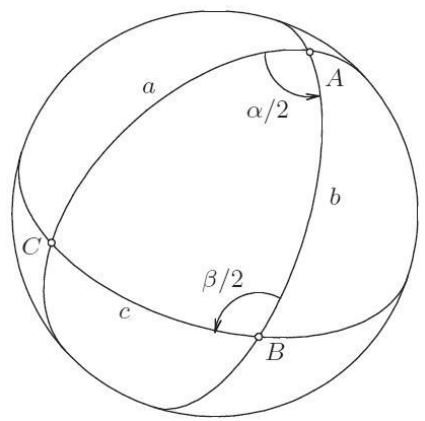

Figure 2: Reflection "lines" on the sphere

The image clearly shows that $\mathcal{R}_{c} \mathcal{R}_{a}$ is rotation (around point $C$ which is the intersection of lines $a$ and $c$ ). The proof of this proposition is completed by previous fact. 


\section{Isometries of sphere and quaternions}

The most elegant (and the most practical) way to describe rotations of $\mathbb{R}^{3}$ or $\mathbb{S}^{2}$ is using quaternions. Quaternion is $2 \times 2$ matrix form

$\boldsymbol{q}=\left(\begin{array}{cc}a+i b & c+i d \\ -c+i d & a-i b\end{array}\right)$, in which $a, b, c, d \in \mathbb{R}$ and $i^{2}=-1$.

The set of all those matrix, i.e. matrix form

where

$$
\mathbb{H}=\{\lambda \mathbf{1}+\alpha \boldsymbol{i}+\beta \boldsymbol{j}+\gamma \boldsymbol{k}: \lambda, \alpha, \beta, \gamma \in \mathbb{R}\},
$$

$$
\mathbf{1}=\left(\begin{array}{ll}
1 & 0 \\
0 & 1
\end{array}\right), \boldsymbol{i}=\left(\begin{array}{cc}
i & 0 \\
0 & -i
\end{array}\right), \boldsymbol{j}=\left(\begin{array}{cc}
0 & 1 \\
-1 & 0
\end{array}\right), \boldsymbol{k}=\left(\begin{array}{ll}
0 & i \\
i & 0
\end{array}\right),
$$

we call Hamilton's algebra or algebra of quaternions over the field $\mathbb{R}$, (see Kalajdžić and Djorić, 2011).

Because $\boldsymbol{q}$ corresponds to the quadruple $(a, b, c, d)$ of real numbers, we can view $\boldsymbol{q}$ as a point in $\mathbb{R}^{4}$. If $\boldsymbol{p}$ is an arbitrary point in $\mathbb{R}^{4}$ then the map sending $\boldsymbol{p} \mapsto \boldsymbol{p} \boldsymbol{q}$ multiplies all distances in $\mathbb{R}^{4}$ by $|\boldsymbol{q}|$, the distance of $\boldsymbol{q}$ from the origin. In particular, if $|\boldsymbol{q}|=1$, then the map $\boldsymbol{p} \mapsto \boldsymbol{p} \boldsymbol{q}$ is an isometry of $\mathbb{R}^{4}$ (Stillwel, 2000).

If $\boldsymbol{p}$ is any quaternion in $(\boldsymbol{i}, \boldsymbol{j}, \boldsymbol{k})$-space,

$$
\boldsymbol{p}=\alpha \boldsymbol{i}+\beta \boldsymbol{j}+\gamma \boldsymbol{k}, \text { where } \alpha, \beta, \gamma \in \mathbb{R},
$$

and if $\boldsymbol{q}$ is any nonzero quaternion, then $\boldsymbol{q} \boldsymbol{p} \boldsymbol{q}^{-1}$ also lies in in $(\boldsymbol{i}, \boldsymbol{j}, \boldsymbol{k})$-space. If $|\boldsymbol{q}|=1$, then the map $\boldsymbol{p} \mapsto \boldsymbol{q} \boldsymbol{p} \boldsymbol{q}^{-1}$ defines an isometry of $\mathbb{R}^{3}$.

Any quaternion with $|\boldsymbol{q}|=1$ can be written in the form

$$
\boldsymbol{q}=\cos \frac{\theta}{2}+(\boldsymbol{i}+m \boldsymbol{j}+n \boldsymbol{k}) \sin \frac{\theta}{2} \text { where } l^{2}+m^{2}+n^{2}=1,
$$

and the isometry $\boldsymbol{p} \mapsto \boldsymbol{q} \boldsymbol{p} \boldsymbol{q}^{-1}$ is a rotation of $(\boldsymbol{i}, \boldsymbol{j}, \boldsymbol{k})$ space through angle $\theta$ about an the axis through $\mathbf{0}$ and $l \boldsymbol{i}+m \boldsymbol{j}+n \boldsymbol{k}$. Rotations of $\mathbb{R}^{3}$ corresponds to the pair of quaternions $\pm \boldsymbol{q}$, with $|\boldsymbol{q}|=1$ (Stillwel, 2000).

By using the Euler's formula we get

$$
\boldsymbol{q}=e^{\frac{\theta}{2}(l \boldsymbol{i}+m \boldsymbol{j}+n \boldsymbol{k})}=\cos \frac{\theta}{2}+(l \boldsymbol{i}+m \boldsymbol{j}+n \boldsymbol{k}) \sin \frac{\theta}{2} .
$$

If we choose the coordinate axes so that they are parallel to $\boldsymbol{i}, \boldsymbol{j}$ and $\boldsymbol{k}$ axis, then rotation axis are practically immediate, and adequate quaternions easy to work with.

Example 1 We can choose $\boldsymbol{i}, \boldsymbol{j}$ and $\boldsymbol{k}$ so they match coordinate axis. For rotation $180^{\circ}$, angle $\theta=\pi$ and therefore $\theta / 2=\pi / 2$. Thus, because $\cos \frac{\pi}{2}=0$ and $\sin \frac{\pi}{2}=1$ rotation for $180^{\circ}$ around $\boldsymbol{i}, \boldsymbol{j}$ and $\boldsymbol{k}$ axis is given by $\boldsymbol{i}, \boldsymbol{j}$ and $\boldsymbol{k}$ quaternions. So, three rotations for $180^{\circ}$ were presented with three pairs of quaternions: $\pm \boldsymbol{i}, \pm \boldsymbol{j}, \pm \boldsymbol{k}$.

According to previous, eight rotations of spheres can be presented with $\frac{1}{\sqrt{3}}( \pm \boldsymbol{i} \pm \boldsymbol{j} \pm \boldsymbol{k})$ (independent choices of + or - sign). 
Factor $\frac{1}{\sqrt{3}}$ is given to absolute value of a quaternion be 1. For each rotation for $120^{\circ}$ we have $\theta= \pm 2 \pi / 3$. So,

$$
\cos \frac{\theta}{2}=\cos \frac{\pi}{3}=\frac{1}{2}, \quad \sin \frac{\theta}{2}= \pm \sin \frac{\pi}{3}= \pm \frac{\sqrt{3}}{2} \text {. }
$$

Considering $\sqrt{3}$ in $\sin \frac{\pi}{3}$ negate factor $\frac{1}{\sqrt{3}}$ in axis of rotation and we see that eight rotation for $120^{\circ}$ are presented by eight pairs of opposite 16 quaternions

$$
\pm \frac{1}{2} \pm \frac{i}{2} \pm \frac{j}{2} \pm \frac{k}{2}
$$

Identically rotation is represented by a pair $\mathbf{\pm} \mathbf{1}$.

\section{Advantages of unit quaternion notation}

There are at least eight methods used fairly commonly to represent rotation, including: orthonormal matrices, axis and angle, Euler angles, Gibbs vector, Pauli spin matrices, Cayley-Klein parameters, Euler or Rodrigues parameters, and Hamiltons quaternions.

One advantage of the unit quaternion representations is that it leads to a clear idea of what the "space of rotations" is; we can observe it as the unit sphere $\mathbb{S}^{3}$ in 4 -space with antipodal points identified ( $\boldsymbol{q}$ represents the same rotation as $\boldsymbol{- q}$ ). It is equivalently in the projective space $\mathbb{P}^{3}$. This makes it possible, for example, to compute averages over all possible attitudes of an object. It also makes it possible to sample the space of rotations in a systematic way, or randomly with uniform sampling density.

Another advantage is that, while redundant (4 numbers to represent 3 degrees of freedom), the extra constraint (namely that it has to be a unit quaternion) is relatively easy to deal with. This makes it possible to find closed-form solutions to some optimization problems involving rotations. Such problems are hard to solve by using orthonormal matrices to represent rotation because of the six non-linear constraints to enforce orthonormality $\left(R^{T} R=I\right)$, and the additional constraint $\operatorname{det}(R)=+1$.

If we compose rotations using multiplication of $3 \times 3$ matrices, numerical problems will conspire to make the results not quite orthonormal. It is difficult to find the "nearest" orthonormal matrix to one that is not quite orthonormal. While multiplying unit quaternions may similarly lead to quaternions that are no longer of unit length, these are easy to normalize.

When it comes to rotating vectors and composing rotations, quaternions may have less of an advantage. While it takes fewer operations to multiply two unit quaternions than it does to multiply two orthonormal matrices, it takes a few more operations to rotate a vector using unit quaternions (although the details depend in both cases on how cleverly the operation is implemented).

\section{Conclusion}

The isometries represent one form of art. Especially interesting are the artistic works in which symmetry dominates. The famous artist, a graphic Mauris Cornelis Escher, has some amazing art work that is based on the Euclidean plane tessellation, so he is a favourite among mathematicians. Tessellation or 
paving of the plane is the process of setting up the geometrical shapes in the plane with no overlaps and gaps. It is possible to generalize tessellation to higher dimensions, and some non-Euclidean geometry also have tilling patterns. Escher spheres which were incurred by paving the sphere with various forms have great importance, and are inspired by Escher paving of the plane. Understanding the isometries of spheres and their synthetic construction provides a deeper understanding of tessellation of sphere (hyperbolic tessellations). The combinations of isometric transformations of sphere lead to various tessellations of the sphere.

Although mathematicians and artists researched and constructed isometries of the hyperbolic plane for years, sphere tessellation have recently been studied closely. One of the most popular methods for the construction sphere tessellation is Goodman-Strauss's method, which is very similar to the method for tessellation of Euclidean plane (see Potter and Ribando, 2004). We usually begin tessellation of the plane by polygon which is mapped using the specific isometric transformation and in that way covering, or "paving" of plane is done. Tessellation of sphere also begins by construction of polygons. Constructed polygon is repeatedly mapped by appropriate isometric transformation so "paving" of sphere expands. Goodman-Strauss construction of tilling of the hyperbolic plane by spherical triangle which is presented 2001. year in the paper Compass and Straightedge in the Poincaré Disk (Goodman-Strauss, 2001) is the construction by using paper and pencil. This construction is much easier to accomplish by using the geometric software, such as CindarellaTM.

Creating of the Gabo curves is another activity that is based on the knowledge of isometries sphere. The key geometric element that occurs in the works of the sculptor Naum Gabo or Robert Engman is very curved, symmetric, closed "path" on the surface of the sphere (Séquin, 2001). Gabo's or Engman's original realization of such paths can be seen as two full waves that are wrapped around the equator of the globe, thereby creating two pairs beams, two of which are almost connected to the north, and the other two at the South Pole, respectively. The simplest Gabo curve is a seam on the tennis balls (see Emmer, 2005). Today, there are software that allow drafting the Gabo curves. However, in order to understand the principle of creation (construction) Gabo curves, it is necessary to have some knowledge about isometries of sphere. Symmetries are especially important. Further research of isometries and their application could go in the direction toward the Gabo curves.

\section{References}

1. Blažić, N. Bokan, N. Lučić, Z. Rakić, Z. (2003). Analytic Geometry (in Serbian). Belgrade: Faculty of Mathematics.

2. Coxeter, H. S. M. (1969). Introduction to Geometry. Hoboken, New Jersey: Wiley Classics Library. 
3. Emmer, M. (2005). The Visual Mind. Massachusetts: The Mit Press Cambridge.

4. Goodman-Strauss, C. (2001). Compass and Straightedge in the Poincaré Disk, The American Mathematical Monthly, 108, 38-49.

5. Kalajdžić, G. Djorić, M. (2011). Geometry (in Serbian). Belgrade: Faculty of Mathematics.

6. Lopandić, D. (2011). Geometry (in Serbian). Belgrade: Zavod za udžbenike.

7. Lučić, Z. (1997). Euclidean and Hyperbolic Geometry (in Serbian). Belgrade: Faculty of Mathematics.

8. Potter, M. Ribando, J. (2004). Isometries, Tessellations and Escher, Oh My! American journal of undergraduate research, 3, 21-28.

9. Séquin, C. H. (2001). Viae Globi - Pathways on a Sphere. Geelong, Australia: Mathematics and Design.

10. Stillwel, J. (2000). The Four Pillars of Geometry. New York: Springer.

Милена Бодановић

Марко Станковић

Марија Јордановић

\section{НЕКИ КОМЕНТАРИ О ИЗОМЕТРИЈАМА СФЕРЕ $\mathbb{S}^{2}$}

Сажетак: Циь овог рада је да покаже да су изометрије сфере рестрикичие изометрија из $\mathbb{R}^{3}$, ито је последица рефлексије великих кругова. У раду је приказан модификован доказ теореме о три рефлексије у $\mathbb{S}^{2}$. Група изометрија cфере $\mathbb{S}^{2}$ је „, веома велика “ ито потврђује и теорема о 2-транзитивности сфере. У раду је изложен доказ ове теореме. Посебно је истакнута група ротащија сфере и показано је да је производ две ротаичје у $\mathbb{S}^{2}$ такође ротаџија. На крају, рад приказује занимљиве примере примена изометрија сфере.

Кључне речи: Изометрија сфере $\mathbb{S}^{2}$, раванска рефлексија, 2-транзитивност сфере, група ротација. 